IZA DP No. 7072

Mobility Across Multiple Generations:

The Iterated Regression Fallacy

Jan Stuhler

December 2012 


\title{
Mobility Across Multiple Generations: The Iterated Regression Fallacy
}

\author{
Jan Stuhler \\ University College London, \\ CReAM and IZA
}

\section{Discussion Paper No. 7072 December 2012}

\author{
IZA \\ P.O. Box 7240 \\ 53072 Bonn \\ Germany \\ Phone: +49-228-3894-0 \\ Fax: +49-228-3894-180 \\ E-mail: iza@iza.org
}

Any opinions expressed here are those of the author(s) and not those of IZA. Research published in this series may include views on policy, but the institute itself takes no institutional policy positions. The IZA research network is committed to the IZA Guiding Principles of Research Integrity.

The Institute for the Study of Labor (IZA) in Bonn is a local and virtual international research center and a place of communication between science, politics and business. IZA is an independent nonprofit organization supported by Deutsche Post Foundation. The center is associated with the University of Bonn and offers a stimulating research environment through its international network, workshops and conferences, data service, project support, research visits and doctoral program. IZA engages in (i) original and internationally competitive research in all fields of labor economics, (ii) development of policy concepts, and (iii) dissemination of research results and concepts to the interested public.

IZA Discussion Papers often represent preliminary work and are circulated to encourage discussion. Citation of such a paper should account for its provisional character. A revised version may be available directly from the author. 
IZA Discussion Paper No. 7072

December 2012

\section{ABSTRACT}

\section{Mobility Across Multiple Generations: The Iterated Regression Fallacy ${ }^{*}$}

Conflicting views about the degree of long-run mobility across multiple generations persist because direct empirical evidence is scarce. Predictions are instead routinely derived by iteration of intergenerational measures, a procedure which implies high long-run mobility even when intergenerational mobility is low. However, the assumption that regression implies perpetual regression is a statistical fallacy. I examine this fallacy, its historical background, and its prevalence. I then present various simple models of intergenerational transmission to consider how the relation between intergenerational and multigenerational mobility is affected by elements of the transmission process. I discuss the role of market luck and indirect transmission; the multiplicity of skills; the role of grandparents; and the causal effect of parental income. The direction of bias depends on modeling assumptions, but elementary properties of the transmission process imply that long-run mobility will likely be lower, possibly much lower, than predictions from intergenerational evidence suggest.

JEL Classification: J62

Keywords: intergenerational mobility, multigenerational mobility, intergenerational income elasticity, regression fallacy

Corresponding author:

Jan Stuhler

University College London

Drayton House Room G13

Gordon Street

London WC1H OAX

United Kingdom

E-mail: j.stuhler@ucl.ac.uk

\footnotetext{
* Financial support from the German National Academic Foundation is gratefully acknowledged. I thank Marco Alfano, Manudeep Bhuller, Anders Björklund, Christian Dustmann, Simon Gorlach, Magne Mogstad, Martin Nybom, Anna Raute, Uta Schönberg, and seminar participants at University College London for valuable comments.
} 


\section{Introduction}

A vast empirical literature has estimated the degree of intergenerational persistence in socio-economic characteristics between parents and their children. There exists however little direct evidence on the degree of long-run mobility across multiple generations, such as between grandparents and their grandchildren. In its absence we rely on extrapolation from available parent-child elasticities. For example, Hertz (2006) reports an intergenerational income elasticity of 0.47 for the United States and proceeds to argue:

"To understand what these statistics mean, consider a rich and a poor family in the United States [...] and ask how much of the difference in the parents' incomes would be transmitted, on average, to their grandchildren. In the United States this would be $(0.47)^{2}$ or 22 percent;"

This procedure - extrapolation by exponentiation - shapes our interpretation of the available intergenerational evidence; it features in policy reports and standard textbooks (Borjas, 2009) as well as in specialized survey articles (Piketty, 2000) and research workshops (Durlauf, 2012).

Persistence in economic status is a central aspect in sociological, economic and political theory. Erikson and Goldthorpe (1992) note that competing theories often contain strong and conflicting hypotheses. Liberal political theories maintain that mobility and the degree of equality of opportunity tend to be high in industrialized societies; and further that high mobility is required to sustain liberal democracies. Marxist theories instead postulate that mobility is low, leading to class struggle and instability. Piketty (2000) notes that conflicting views also feature prominently in economic writings. But persistence is not only a central aspect in competing schools of thoughts, it matters also on a practical level. We may for example wonder if specific social policies mask inequalities between families only temporarily or if they have lasting effects on their relative fortunes.

Conflicting views about the degree of long-run mobility persist because we lack empirical evidence. Our knowledge about intergenerational mobility on the other hand has advanced greatly in the last two decades. The finding that income mobility is much lower than previously believed, and particularly low in countries with high levels of cross-sectional inequality in which it is more consequential (such as the United States or United Kingdom), has been received with some concern. But the standard extrapolation procedure provides ammunition for a contrarian standpoint that disputes the significance of those findings, as it implies high long-run mobility even when parent-child mobility is low (see for example Mankiw, 2006).

The idea that exponentiation of the intergenerational elasticity can approximate long-run persistence of economic status appears already in Becker and Tomes' classic work. In section IV of Becker and Tomes (1979) they draw attention to special cases of their theoretical model that seem to vindicate the exponentiation procedure, illustrating its implications by quoting the old proverb "from shirtsleeves to shirtsleeves in four generations". But the same section also contains simulated paths of income for the full model, which clarify that those implications do not generally hold. Puzzlingly, this dependence on theoretical assumptions is disregarded in Becker and Tomes' later work. In section V of Becker and Tomes (1986) they recite a bolder version of the shirtsleeves-proverb ( "... in three generations"), this time not with reference to a special theoretical case but applied without reservations on intergenerational elasticity estimates from the empirical literature. In particular they exponentiate one of those elasticities to argue that the prevalence of poverty in a family tends to disappear within few generations. ${ }^{1}$

\footnotetext{
${ }^{1}$ Their trust in this conclusion becomes apparent in their response to a seemingly glaring contradiction, namely the observation that some groups, such as black Americans, suffer from persistent economic disadvantage. Instead of reconsidering
} 
But the assumption underlying this conclusion, that regression implies perpetual regression, is a statistical fallacy. In this note I present various simple models of intergenerational transmission to illustrate this fallacy and to provide a theoretical base for the emerging empirical literature on multigenerational persistence. Starting from a baseline model I successively consider how various elements of the transmission process affect the relationship between intergenerational and multigenerational mobility, discussing the role of market luck and indirect transmission; the multiplicity of skills; the role of grandparents; and finally the causal effect of parental income or status. I find that the direction of bias depends in principle on modeling assumptions, but elementary properties of the intergenerational transmission process imply that long-run mobility will likely be lower, and possibly much lower, than the standard extrapolation procedure implies.

\section{The Iterated Regression Fallacy}

The intergenerational income elasticity between parents and their children is defined as the OLS slope coefficient from a regression of $\log$ lifetime income of the offspring generation $t$ of family $i, y_{i t}$, on parental log lifetime income $y_{i t-1}$,

$$
y_{i t}=\beta_{-1} y_{i t-1}+\varepsilon_{i t} .
$$

The elasticity measures the percentage differential in expected offspring income with respect to a percentage differential in parental income; a high elasticity represents low mobility. But how does this parent-child elasticity compare with the elasticity across three or more generations, e.g. between grandparents and their grandchildren? The idea that the latter equals the square of the former, so that persistence declines geometrically, may appear as a natural consequence of regression: if $\beta_{-1}$ measures to what degree parental deviations from the mean are passed to their children then surely $\left(\beta_{-1}\right)^{2}$ measures their extent after being passed twice from parents to children? Formally, one may believe that equation (1) can be used to rewrite the grandparent-grandchild elasticity $\beta_{-2}$ as

$$
\beta_{-2} \equiv \frac{\operatorname{Cov}\left(y_{i t}, y_{i t-2}\right)}{\operatorname{Var}\left(y_{i t-2}\right)}=\frac{\operatorname{Cov}\left(\beta_{-1} y_{i t-1}+\varepsilon_{i t}, y_{i t-2}\right)}{\operatorname{Var}\left(y_{i t-2}\right)}=\left(\beta_{-1}\right)^{2} .
$$

The error lies in the last step. The interpretation of equation (1) itself may be the source of confusion. The equation has no structural interpretation, nor does it represent an AR(1) process. It captures instead a statistical relationship: the component $\beta_{-1} y_{i t-1}$ is the best linear predictor (in a MMSE sense) of $y_{i t}$ since $\beta_{-1}$ is defined to be the OLS slope coefficient. While $\varepsilon_{i t}$ is thus by construction uncorrelated to parental income $y_{i t-1}$, it is not necessarily uncorrelated with grandparental income $y_{i t-2}$. The equation does not represent a data generating process from which predictions beyond two generations could be derived; it is misspecified for these purposes.

The underlying fallacy, the belief that regression toward the mean between two observations implies perpetual regression across observations, appears to be a classic one. Galton himself fell fault of it, as noted by Bulmer (2003). It is comprehensively discussed in Nesselroade et al. (1980) under the caption "expectation fallacy". ${ }^{2}$ That name seems not to have caught on; it may also be too unspecific in economics, where the term "expectation" relates not only to statistical but also behavioral concepts. In this note I instead use the name "iterated regression fallacy", which is hopefully evocative of Galton's work, the intergenerational context, and other classic regression fallacies. ${ }^{3}$ We can formulate a simple theoretical model to examine it in the context of the intergenerational mobility literature.

their procedure for extrapolation from intergenerational equations, Becker and Tomes (1986) argue that those equations could differ for Blacks, for example because discrimination affects intercepts (p. 10) or slope coefficients (p. 28).

${ }^{2}$ The name "iterated expectation fallacy" would be also fitting, as it can be viewed as an incorrect application of the law of iterated expectations: extrapolation by exponentiation would be reasonable if $E\left[y_{i t} \mid y_{i t-2}\right]=E\left[E\left[y_{i t} \mid y_{i t-1}\right] \mid y_{i t-2}\right]$, which however only holds if $y_{i t}$ follows a Markov process.

${ }^{3}$ Such as the fallacy that regression towards the mean implies convergence to the mean, see Friedman (1992); or the failure to account for regression to the mean in comparisons over time (Jerrim and Vignoles, 2012, discuss a recent example). 
A simple model. Consider a simplified one-parent one-offspring family structure for which income and intergenerational transmission are governed by

$$
\begin{aligned}
& y_{i t}=\rho e_{i t}+u_{i t} \\
& e_{i t}=\lambda e_{i t-1}+v_{i t},
\end{aligned}
$$

in which $y_{i t}, \log$ lifetime income in generation $t$ of family $i$, depends on human capital $e_{i t}$ (according to returns $\rho$ ) that is partially inherited within families (according to heritability $\lambda$ ). I use the term heritability here in a wide sense, representing not only genetic but also other causal pathways of transmission from parents to children (e.g. parental upbringing). The noise terms $u_{i t}$ and $v_{i t}$ represent market and endowment luck, and are assumed to be uncorrelated with each other and past values. To simplify the presentation assume throughout that variables are measured as trendless indices with mean zero and variance one, such that slope parameters can be interpreted as correlations; and further that those indices measure favorable traits that are not negatively correlated within families, such that all parameters are non-negative. Note that the parameter $\rho$ then measures the transferability of human capital into income; for example, $\rho=1$ implies that differences in incomes are fully explained by individuals' own characteristics. The $i$ subscript is dropped in the subsequent analysis.

Given equations (2) and (3), and the assumption that all variances are unity, the parent-child elasticity equals

$$
\begin{aligned}
\beta_{-1} & =\operatorname{Cov}\left(y_{t}, y_{t-1}\right) \\
& =\rho^{2} \lambda,
\end{aligned}
$$

and the elasticity across three generations instead equals

$$
\begin{aligned}
\beta_{-2} & =\operatorname{Cov}\left(y_{t}, y_{t-2}\right) \\
& =\rho^{2} \lambda^{2} .
\end{aligned}
$$

The extrapolation error from exponentiation of the parent-child elasticity equals

$$
\begin{aligned}
\Delta & =\left(\beta_{-1}\right)^{2}-\beta_{-2} \\
& =\left(\rho^{2}-1\right) \rho^{2} \lambda^{2},
\end{aligned}
$$

which is negative for $0<\rho<1$ and $0<\lambda<1$, that is as long as the intergenerational transmission of human capital and its transformation into income are not perfect. ${ }^{4}$ The extrapolation error $\Delta$ will be large when $\rho$ is small relative to the degree of heritability captured by $\lambda$. Exponentiation of the parentchild elasticity does therefore not recover multigenerational elasticities, and may instead provide a very misleading picture. But why do extrapolations from $\beta_{-1}$ overstate long-run mobility? The answer may become obvious when we consider the existence of an additional layer in the transmission process.

An additional layer. Assume that human capital is not directly transmitted within families, but that parents bequeath certain traits $a_{t}$ (according to heritability $\pi$ ), which in turn affect human capital $e_{t}$ (according to transferability $\mu$ ). ${ }^{5}$ Intergenerational transmission is then governed by

$$
\begin{aligned}
& y_{t}=\rho e_{t}+u_{t} \\
& e_{t}=\mu a_{t}+v_{t} \\
& a_{t}=\pi a_{t-1}+w_{t} .
\end{aligned}
$$

\footnotetext{
${ }^{4}$ These and subsequent results do not rely on the simplifying assumption that parameter values are stable; the product of intergenerational elasticities will underestimate multigenerational persistence also when those elasticities vary across generations.

${ }^{5}$ One may assign more specific interpretations for each layer (e.g. that the lowest layer represents genetic transmission) and include additional explanatory variable, such as in Conlisk (1974). My interest here is only to capture the idea that income transmission may occur rather indirectly.
} 
The parent-child elasticity equals

$$
\beta_{-1}=\rho^{2} \mu^{2} \pi
$$

and the grandparent-grandchild elasticity equals

$$
\beta_{-2}=\rho^{2} \mu^{2} \pi^{2}
$$

Consider parameterizations for $\mu$ and $\pi$ that yield the same parent-child elasticity as the two-layers model, which requires $\lambda=\mu^{2} \pi$. The extrapolation error then equals

$$
\Delta=\left(\rho^{2}-\frac{1}{\mu^{2}}\right) \rho^{2} \lambda^{2}
$$

Comparison to equation (6) reveals that the extrapolation error will be larger in the three-layers model iff $\mu<1$; the introduction of an additional layer in the transmission process raises the gap between extrapolated and actual long-run mobility.

These results stems from the interplay between mechanisms of transmission between and within generations. Both imperfect inheritability of traits and their imperfect transferability into income $(\rho<1)$ decrease parent-child persistence of economic status. But regression to the mean beyond two generations depends only on the heritability parameter. For example, persistence equals $\beta_{-2}=\beta_{-1} \pi$ across three generations, $\beta_{-3}=\beta_{-1} \pi^{2}$ across four generations, and so on. The intuition is simple: traits are inherited multiple times, but for each generation they are only once priced and transformed into income.

The extrapolation error will be substantial even if the role of market luck is modest. For example, exponentiation of an intergenerational elasticity of 0.5 implies $\left\{\beta_{-1}, \beta_{-2}, \beta_{-3}\right\}=\{0.5,0.25,0.125\}$ and thus rapid regression to the mean. Now consider the actual elasticities implied by the two-layers model, from equation (4) and (5). If $\rho=0.9$ (such that factors orthogonal to individual abilities explain about a fifth of the cross-sectional variance in income) we have $\lambda \approx 0.62$ and the elasticities equal $\{0.5,0.31,0.19\}$. If $\rho=0.8$ (market luck explains about a third) $\lambda \approx 0.78$ and the elasticities equal $\{0.5,0.39,0.31\}$, implying substantially higher persistence of economic status within families.

The underlying assumptions should be uncontroversial. We do not expect individuals with equivalent levels of human capital to have exactly equal incomes, as factors outside of individual control drive a wedge between their traits and incomes. ${ }^{6}$ We also know that at least part of the intergenerational transmission of income occurs via indirect mechanisms, for example through genetic inheritance or parental upbringing.

Various implications follow. First, long-run mobility will be smaller the more intergenerational mobility is attributable to imperfect transferability than low heritability of endowments. This will tend to be the case if intergenerational transmission is more indirect. Second, we may worry that certain policies mask inequalities only temporarily. For example, comprehensive schooling may reduce the degree to which differences in child abilities lead to differences in human capital (a reduction of $\mu$ in the three-layers model) and thus increase intergenerational mobility, but it may not increase long-run mobility much if the inheritability of abilities does not change. Third, if cross-country differences in intergenerational mobility extend to the long run depends crucially on if those differences are due to variation in the heritability or transferability of endowments across countries. For example, Nordic countries will be characterized by exceptional long-run mobility if their high levels of intergenerational mobility are caused by policies and institutions that decrease the heritability of traits, less so if they are due to policies that interfere with the formation of market prices for those traits.

\footnotetext{
${ }^{6}$ This wedge may be sizable - earnings regressions only explain a fraction of the variation in the dependent variable, even when the list of regressors is large (e.g. Zax and Rees, 2002); monozygotic twins have substantially different earnings even while their genetic inheritance and early family background are very similar; and various economic literatures show that events outside of individual control (such as occupation-, region-, or firm-specific demand shocks) affect incomes.
} 
An additional factor. But there exists a second elementary reason why multigenerational persistence of economic status will tend to be higher than extrapolations from intergenerational elasticities imply. Introduce a second factor into our starting model,

$$
\begin{aligned}
y_{t} & =\rho_{1} e_{1 t}+\rho_{2} e_{2 t}+u_{t} \\
e_{1 t} & =\lambda_{1} e_{1, t-1}+v_{1 t} \\
e_{2 t} & =\lambda_{2} e_{2, t-1}+v_{2 t},
\end{aligned}
$$

assuming that two traits are inherited from parents according to heritability parameters $\lambda_{1}$ and $\lambda_{2}$. For simplicity also assume that the endowment luck terms $v_{1 t}$ and $v_{2 t}$ are uncorrelated, such that $\operatorname{Cov}\left(e_{1 t}, e_{2 t}\right)=0 \forall t$. Assume further that both traits play a role in the determination of incomes, such that $0<\rho_{1}<1$ and $0<\rho_{2}<1$. The parent-child elasticity then equals

$$
\beta_{-1}=\rho_{1}^{2} \lambda_{1}+\rho_{2}^{2} \lambda_{2},
$$

and the grandparent-grandchild elasticity equals

$$
\beta_{-2}=\rho_{1}^{2} \lambda_{1}^{2}+\rho_{2}^{2} \lambda_{2}^{2}
$$

The extrapolation error equals

$$
\Delta=\left(\rho_{1}^{2}-1\right) \rho_{1}^{2} \lambda_{1}^{2}+\left(\rho_{2}^{2}-1\right) \rho_{2}^{2} \lambda_{2}^{2}+2 \rho_{1}^{2} \rho_{2}^{2} \lambda_{1} \lambda_{2} .
$$

Assume for a moment that incomes are indeed perfectly determined by individual traits, such that $\rho_{1}^{2}+\rho_{2}^{2}=1$ and $\operatorname{Var}\left(u_{t}\right)=0$. Equation (18) can then be written as

$$
\Delta=\rho_{1}^{2}\left(\rho_{1}^{2}-1\right)\left(\lambda_{1}-\lambda_{2}\right)^{2} .
$$

This expression is negative for $\lambda_{1} \neq \lambda_{2}$. In contrast to the previous models, exponentiated parentchild elasticities understate multigenerational persistence even when human capital determines incomes perfectly, as long as those traits that constitute human capital are not all equally strong inherited within families. This result can be understood as the application of Jensen's inequality on the case of intergenerational transmission: the square of the average heritability across traits is smaller than the average of the square of those heritabilities. Inequality between families declines therefore more slowly if intergenerational persistence stems from multiple causal pathways.

Highly inheritable traits (such as IQ) explain an increasing share of the long-run persistence in income. In particular, multigenerational elasticities will never converge to zero if any characteristic is perfectly transmitted. For example, physical traits such as skin color may be highly persistent in multiethnic societies if interracial marriage is rare, and may lead to persistent disadvantage of families if groups are discriminated on the labor market. For the analysis of long-run mobility it is thus essential to look beyond scalar models, even if those models have proved to be useful for other questions in the literature.

This applies in particular to the framework presented in Becker and Tomes (1979), which still underlies much of the theoretical work in the literature. As it contains only a scalar measure of human capital it cannot capture implications that stem from the existence of multiple transmission mechanisms. Becker and Tomes also emphasize market efficiency, and the noise term that represents income luck is eliminated altogether in descendants of their model such as Solon (2004), where human capital is assumed to transform perfectly into income. I showed that both these simplifications lead us to underestimate long-run persistence. ${ }^{7}$

\footnotetext{
${ }^{7}$ These findings support arguments made by Goldberger (1989), who notes that an explicit consideration of utility maximization behavior of parents (as in Becker and Tomes, 1979) to motivate "mechanical" transmission equations may not provide additional implications and may then distract from the assumed properties of those equations. The Becker and Tomes model in general and its modified descendants in particular lead to transmission equations that are much simplified compared to earlier models in the literature, which did contain noise terms to capture market luck and multiple inheritance mechanisms (e.g. Conlisk, 1969).
} 
An additional generation. These results do not rely on the existence of independent higher-order causal effects, such as from grandparents on their grandchildren. I assumed throughout that the intergenerational transmission process has a memory of only one generation. From the observation that $\left(\beta_{-1}\right)^{2}<\beta_{-2}$ we can therefore not conclude, as for example Lindahl et al. (2012) do, that the intergenerational transmission process has a memory of more than one generation. The intuition that such higher-order effects raise long-run persistence is however correct. To see this assume that offspring human capital depends on both parents and grandparents, such that equation (3) becomes

$$
e_{t}=\lambda_{-1} e_{t-1}+\lambda_{-2} e_{t-2}+v_{t}
$$

with $\lambda_{-2}>0$. Assuming stationarity the parent-child elasticity equals

$$
\beta_{-1}=\rho^{2}\left(\frac{\lambda_{-1}}{1-\lambda_{-2}}\right)
$$

Consider parameterizations that yield the same intergenerational elasticity as the previous model, such that $\lambda=\lambda_{-1} /\left(1-\lambda_{-2}\right)$. The grandparent-grandchild elasticity,

$$
\beta_{-2}=\rho^{2} \lambda^{2}+\rho^{2} \lambda_{-2}\left(1-\lambda^{2}\right),
$$

is then greater than the respective elasticity in the baseline model (assuming $\rho>0$ and $\lambda<1$ ). This simple example does not illustrate the various ways how grandparents may influence their grandchildren, but it illustrates that such influence strengthens multigenerational relative to intergenerational persistence. ${ }^{8}$

Parental investment. All previous results point to "excess persistence", to the conclusion that extrapolated intergenerational elasticities understate long-run persistence. But we can certainly think of circumstances in which the opposite holds, for which I will give one example.

Assume that parental income or economic status have a causal effect on offspring; for example indirectly through parental investments in offspring human capital, or more directly through reputation or networking effects on the labor market. Consider the first case, such that equations (2) and (3) change into

$$
\begin{aligned}
& y_{t}=\rho e_{t}+u_{t} \\
& e_{t}=\theta y_{t-1}+\eta e_{t-1}+v_{t} .
\end{aligned}
$$

The parent-child and grandparent-grandchild elasticities then equal

$$
\begin{aligned}
& \beta_{-1}=\rho \theta+\rho^{2} \eta \\
& \beta_{-2}=\left(\rho \eta+\rho^{2} \theta\right)(\rho \eta+\theta) .
\end{aligned}
$$

Consider again parameterizations that yield the same level of $\beta_{-1}$, which requires $\eta<\lambda$ (assuming $\rho>0$ and $\theta>0$ ). The extrapolation error,

$$
\Delta=\left(\rho^{2}-1\right) \eta \beta_{-1},
$$

is smaller than the error in our first model (which equals $\left.\left(\rho^{2}-1\right) \lambda \beta_{-1}\right)$, but it will still be negative. Our previous findings thus hold also when parental income and investment affect offspring human capital.

\footnotetext{
${ }^{8}$ Mare (2011) suggests various mechanisms that could give rise to multigenerational effects and also touches on the relationship between intergenerational and multigenerational measures of persistence.
} 
Now instead assume that parental income has a direct effect on offspring income that is independent of offspring characteristics, such that equations (2) and (3) change into

$$
\begin{aligned}
& y_{t}=\phi y_{t-1}+\tau e_{t}+u_{t} \\
& e_{t}=\lambda e_{t-1}+v_{t} .
\end{aligned}
$$

The parent-child and grandparent-grandchild elasticities then equal

$$
\begin{aligned}
& \beta_{-1}=\phi+\frac{\tau^{2} \lambda}{1-\phi \lambda} \\
& \beta_{-2}=\phi^{2}+\frac{\tau^{2} \lambda}{1-\phi \lambda}(\phi+\lambda),
\end{aligned}
$$

The extrapolation error equals

$$
\Delta=\left(\frac{\tau^{2} \lambda}{1-\phi \lambda}\right)^{2}+(\phi-\lambda) \frac{\tau^{2} \lambda}{1-\phi \lambda} .
$$

which in contrast to the previous examples may be positive, for example if $\phi>\lambda$. In this model, income is affected by both parental income and ability, but offspring ability is affected exclusively by parental ability. While the intergenerational persistence of economic status may be strongly affected by the direct effect of income $\phi$, in the long run persistence will be dominated by the heritability of ability $\lambda$. If the former is large and the latter is small we have a system in which multigenerational persistence is weaker than exponentiation of $\beta_{-1}$ implies.

These two models illustrate that different beliefs about causal pathways of transmission are consistent with different expectations about long-run mobility; one cannot have it both ways. For example, if one believes that children from affluent families tend to fare better mainly because inherited traits and parental investment raise their productive abilities then one should also expect that long-run mobility is lower than exponentiated intergenerational elasticities imply. But genetic inheritance and parental investment are mechanisms emphasized on the right wing of the political spectrum, in which the significance of low intergenerational mobility estimates is challenged precisely by the argument that they nevertheless imply high long-run mobility (see Mankiw, 2006). The opposite argument applies if one believes that intergenerational persistence of income is predominantly due to mechanisms unrelated to individual productivity, such as nepotism. For example, if income persistence is only due to the direct influence of parental income then its decline over generations is indeed geometrically, and even low levels of intergenerational mobility would imply rapid multigenerational regression to the mean. One side's favourite causal explanations for intergenerational correlations thus serve inadvertently as an implicit endorsement of the other side's beliefs about the extent of long-run mobility.

\section{Conclusions}

I analyzed the relationship between intergenerational and long-run mobility, and illustrated how its properties depend on the nature of transmission between parents and children. Questions on mobility across multiple generations are thus closely related to existing work on the causal pathways of transmission. This relation is interesting in both ways: I noted what implications different transmission processes have for multigenerational mobility, but one could turn the question around to consider how a comparison of parent-child and grandparent-child elasticities may identify features of the causal process.

Most of the discussion implies that the standard practice of extrapolating from intergenerational elasticities understates long-run persistence in economic status. But that conclusion may appear frag-

ile, as I showed that one can find structures (functional form and parameter assumptions) that lead 
to the opposite conclusion. Can we then only conclude that naive extrapolations of intergenerational elasticities are misleading - the iterated regression fallacy - or can we also agree in which direction we expect them to be biased? I believe the latter is possible if we take the extensive work on transmission mechanisms and determinants of income into account, as it places restrictions on the properties of admissible structures.

I discussed the role of market luck and indirect transmission; of the multiplicity of skills and causal pathways; of other ancestors such as grandparents; and of the causal effect of parental income or status. I find that even small amounts of market luck (of factors that are orthogonal to individual abilities) imply much less rapid multigenerational regression to the mean for a given level of intergenerational mobility. I also noted why the role of market luck may be not negligible. Multiplicity of skills also matters, as regression to the mean tends to slow down across generations if intergenerational persistence is a product of various causal pathways. It will certainly decelerate if earlier ancestors such as grandparents or their outcomes have an independent causal effect on offspring. Finally, we have evidence that the causal effect of parental income is small (see Björklund and Jäntti, 2009), and we may expect that at least part of this effect works indirectly (e.g. through parental investments in child human capital). Together these arguments imply that long-run persistence is higher, maybe much higher than the standard interpretation of intergenerational elasticities implies.

This prediction and its underlying arguments also apply to mobility in other socio-economic outcomes, such as educational attainment. Income has however an additional layer of complexity from the role of prices, which constitute a source for imperfect correlation between inherited characteristics and income. Extrapolations of income elasticities may thus tend to be more misleading than extrapolations of elasticities of other socio-economic characteristics.

Data that allow estimation on mobility beyond two generations, such as between grandparents and their grandchildren, are as yet scarce. But a number of promising empirical studies are in the making. Some of the first available evidence does support the argument that multigenerational persistence is higher than previously thought: Lindahl et al. (2012) find that persistence in education and income over three Swedish generations is severely underpredicted by measures of intergenerational persistence. Income elasticities are difficult to estimate even in the intergenerational case, as approximation of lifetime income based on sparse data requires an understanding of income dynamics over the lifecycle. But their findings are supported by Clark and Cummins (2012), who measures social status by other socio-economic outcomes, finding high persistence in England over very long time intervals. 


\section{References}

Becker, G., And N. Tomes (1979): "An Equilibrium Theory of the Distribution of Income and Intergenerational Mobility,” The Journal of Political Economy, 87(6), 1153-1189.

(1986): "Human Capital and the Rise and Fall of Families," Journal of Labor Economics, 4(3), 1-39.

BJÖRKLUND, A., AND M. JÄNTTI (2009): “Intergenerational Income Mobility and the Role of Family Background," in Oxford Handbook of Economic Inequality, ed. by B. N. Salverda Wiemer, and T. M. Smeeding. Oxford University Press.

BoRJAS, G. J. (2009): Labor Economics. New York: Irwin/McGraw-Hill, 5th edn.

Bulmer, M. (2003): Francis Galton: Pioneer of Heredity and Biometry. Johns Hopkins University Press.

Clark, G., And N. Cummins (2012): "What is the True Rate of Social Mobility? Surnames and Social Mobility, England 1800-2012,” Discussion paper, Unpublished working paper.

CONLISK, J. (1969): "An Approach to the Theory of Inequality in the Size Distribution of Income," Economic Inquiry, 7(2), 180-186.

- (1974): “Can Equalization of Opportunity Reduce Social Mobility?," The American Economic Review, 64(1), pp. 80-90.

Durlauf, S. (2012): “Intergenerational Mobility: Introductory Comments," in Summer School on Socioeconomic Inequality, University of Chicago, 2012. https://ineqss.uchicago.edu/.

ERIKSOn, R., And J. GoldThORPe (1992): The Constant Flux: A Study of Class Mobility in Industrial Societies. Oxford University Press, USA.

FRIEDMAN, M. (1992): “Do Old Fallacies Ever Die?,” Journal of Economic Literature, 30(4), 21292132.

Goldberger, A. S. (1989): "Economic and Mechanical Models of Intergenerational Transmission," The American Economic Review, 79(3), pp. 504-513.

Hertz, T. (2006): "Understanding Mobility in America," in Report For the Center for American Progress.

Jerrim, J., And A. Vignoles (2012): "Social Mobility, Regression to the Mean and the Cognitive Development of High Ability Children From Disadvantaged Homes," Journal of the Royal Statistical Society: Series A.

Lindahl, M., M. Palme, S. Sandgren Massih, And A. Sjögren (2012): “The Intergenerational Persistence of Human Capital: An Empirical Analysis of Four Generations," IZA Discussion Papers 6463, Institute for the Study of Labor (IZA).

Mankiw, G. (2006): “Rich Dad, Poor Dad," in Greg Mankiw's Blog, published on April 27, 2006. http://gregmankiw.blogspot.co.uk/2006/04/rich-dad-poor-dad.html.

Mare, R. (2011): “A Multigenerational View of Inequality,” Demography, 48(1), 1-23.

Nesselroade, J. R., S. M. Stigler, and P. B. Baltes (1980): "Regression Toward the Mean and the Study of Change," Psychological Bulletin, 88(3). 
PIKeTty, T. (2000): “Theories of Persistent Inequality and Intergenerational Mobility," in Handbook of Income Distribution, ed. by A. B. Atkinson, and F. Bourguignon, vol. 1, pp. 429 - 476. Elsevier.

Solon, G. (2004): “A Model of Intergenerational Mobility Variation over Time and Place," Generational Income Mobility in North America and Europe, pp. 38-47.

ZAX, J., AND D. ReES (2002): "IQ, Academic Performance, Environment, and Earnings," Review of Economics and Statistics, 84(4), 600-616. 